Linguistique, littérature, didactique

151-152 | 2011

Anthropologies de la littérature

\title{
Les enjeux passés et futurs de l'imaginaire
}

Mythème, mythanalyse et mythocritique

\section{Philippe Walter}

\section{(2) OpenEdition \\ Journals}

Édition électronique

URL : http://journals.openedition.org/pratiques/1769

DOI : 10.4000/pratiques. 1769

ISSN : 2425-2042

Éditeur

Centre de recherche sur les médiations (CREM)

Édition imprimée

Date de publication : 15 décembre 2011

Pagination : 39-48

Référence électronique

Philippe Walter, «Les enjeux passés et futurs de l'imaginaire », Pratiques [En ligne], 151-152 | 2011, mis en ligne le 13 juin 2014, consulté le 19 avril 2019. URL : http://journals.openedition.org/pratiques/1769 ; DOI : 10.4000/pratiques. 1769

() Tous droits réservés 


\title{
Les enjeux passés et futurs de l'imaginaire Mythème, mythanalyse et mythocritique
}

\author{
Philippe Walter \\ Université de Grenoble, Directeur du CRI (EA 610)
}

La fondation du Centre de Recherche sur l'Imaginaire (CRI) à Grenoble, en 1966, marqua une étape décisive dans la formation d'un «nouvel esprit anthropologique». Depuis sa création, le CRI a trouvé son unité et sa cohérence dans la réflexion sur l'imaginaire et l'imagination symbolique en dégageant les aspects, l'évolution et le sens d'une herméneutique des images et des mythes dans l'imaginaire d'une culture, d'une époque ou d'un créateur.

La méthode d'analyse privilégie les procédures symboliques (symboles, mythes, images) comme éléments déterminants de la création littéraire et artistique soustendant, sur une période donnée, les attitudes socio-historico-culturelles. Pour ces études, sont mobilisées, à des degrés divers mais permanents, la psychologie, la sociologie, l'anthropologie, l'ethnologie, la philosophie et la critique littéraire, mais aussi l'histoire des religions, la linguistique, les sciences de la communication et, entre autres, la mythologie, dans un souci de croisement raisonné des disciplines. Le regard sur certains concepts émergents de l'imaginaire permet de mesurer les évolutions de ces recherches sur plusieurs décennies. On privilégiera celui de mythème.

\section{Au commencement était le mythème}

Introduit dans le champ théorique par l'anthropologue Claude Lévi-Strauss, le concept de mythème a été repris et développé par Gilbert Durand qui en a fait l'unité significative minimale du mythe, son principe même d'identification ainsi que l'instrument de son interprétation. Il est utile de rapporter l'histoire de ce concept de base en mythanalyse. Il fut à l'origine d'une reconnaissance de «l'a-logique du mythe », c'est-à-dire d'une capacité de l'imaginaire à exister (et à signifier) dans des formes observables mais autrement que le langage ordinaire. 
Dès 1945, Cl. Lévi-Strauss avait défini les bases de la mythanalyse dans un article intitulé «L'analyse structurale en linguistique et en anthropologie » ${ }^{(1)}$. Fortement marqué par la linguistique structurale de Roman Jakobson, il y mettait notamment en lumière la notion de structure dans ses rapports avec celle de relation. Cet article repris en 1958 dans Anthropologie structurale pose « la différence entre [les] deux notions ». Constatant que « la notion de structure sociale ne se rapporte pas à la réalité empirique, mais aux modèles construits d'après celle-ci », il en conclut que « les relations sociales sont la matière première employée pour la construction des modèles qui rendent manifeste la structure sociale elle-même ». Parmi ces modèles, il faut évidemment ranger toutes les productions de l'imaginaire (mythes, littératures, œuvres d'art, etc.)

L'étude des mythes, notamment amérindiens, permit à Lévi-Strauss d'illustrer et d'approfondir une méthode qu'il présenta dès 1958 dans un chapitre d'Anthropologie structurale intitulé «La structure des mythes » ${ }^{(2)}$. A la question du sens du mythe, il répond : «Si les mythes ont un sens, celui-ci ne peut tenir aux éléments isolés qui entrent dans leur composition, mais à la manière dont ces éléments se trouvent combinés ». C'est l'analyse linguistique structurale que se donne alors Lévi-Strauss comme modèle théorique. Posant que «le mythe est langage ", même s'il s'agit d' " un langage qui travaille à un niveau très élevé », il affirme que, comme toute réalité linguistique, "le mythe est formé d'unités constitutives ». Non seulement ces unités occupent dans la structure du mythe la même place que les phonèmes, morphèmes ou sémantèmes dans la structure de la langue mais de plus elles s'articulent, comme leurs modèles linguistiques, en structures à complexité croissante à savoir les mythèmes, nom donné par Lévi-Strauss aux «grosses unités constitutives » qui, seront donc à "chercher au niveau de la phrase » et constitueront les éléments les plus complexes spécifiques au mythe.

S'appuyant sur l'analyse du mythe d'Edipe, Lévi-Strauss, dans un tableau en quatre colonnes, fait apparaître la structure de ses mythèmes, c'est-à-dire de courtes phrases illustrant une « relation », celle qu'un sujet entretient avec un prédicat, du type : «Cadmos tue le dragon » ou « Edipe : «pied enflé ». Cette structure à la fois synchronique, récurrente (lecture du tableau par colonne, de haut en bas, faisant apparaître des séries de mythèmes en écho) et diachronique (lecture du tableau de gauche à droite, ligne à ligne permettant de retrouver la temporalité historique du récit) met en évidence que le mythème isolé ne peut pas rendre compte de la dynamique mythique ; cette dernière, pour apparaître et devenir signifiante, doit se réfléchir à travers des ensembles de relations : "Nous posons, en effet, que les véritables unités constitutives du mythe ne sont pas les relations isolées, mais des paquets de relations, et que c'est seulement sous forme de combinaisons de tels paquets que les unités constitutives acquièrent un fonction signifiante ». Une idée forte se dégage de cette lecture tabulaire : un mythe ne se lit pas seulement de manière syntagmatique mais il se lit aussi sur le plan paradigmatique ; il suppose en quelque façon, pour sa compréhension, une dépliement du discours ${ }^{(3)}$.

(1) Cl. Lévi-Strauss, «L'analyse structurale en linguistique et en anthropologie », Anthropologie structurale, Paris, Plon, 1958, pp. 43-69 (1 $1^{\text {re }}$ éd., Word, Journal of the Linguistic Circle of New York, vol. 1, n² ; August 1945, pp. 1-21; rééd. Agora/Pocket, 1974). Sur le mythème, Anthropologie structurale, op. cit., pp. 241-246.

(2) Cl. Lévi-Strauss, « La structure des mythes », op. cit., pp. 235-265.

(3) Voir un autre exemple de ce type d'analyse dans C. Lévi-Strauss, « La geste d'Asdiwal », $A n$ thropologie structurale deux, Paris, Plon, 1973 (rééd. Plon, Agora/Pocket, 1996), pp. 175-233. 
Comme le souligne Lévi-Strauss, ce dispositif sert non pas à raconter le mythe mais à le comprendre ; ce que mettent en lumière chacune des quatre colonnes et les colonnes entre elles, ce sont des relations de similitude ou d'opposition qui, puisées à diverses étapes diégétiques du récit et regroupées artificiellement, révèlent structure et sens du mythe à travers ses mythèmes. Ainsi la fin première du mythe d'Edipe pourrait bien être de surmonter, d'affronter ce qu'une société ressent comme étant de l'ordre de l'insurmontable. Si la troisième colonne du tableau qui voit la victoire de l'homme sur les monstres met en évidence « la négation de l'autochtonie de l'homme ", la quatrième colonne qui illustre la permanence du handicap physique pour les hommes nés de la terre, insiste quant à elle sur « la persistance de l'autochtonie de l'homme ». De cette façon, pour Lévi-Strauss, une société semble s'être donné par l'intermédiaire du mythe un « instrument logique » pour signifier une aporie fondatrice. Ainsi, les mythèmes, leur jeu de récurrences, leur présence répétitive constituent bien " la matière première employée pour la construction des modèles qui rendent manifeste la structure sociale elle-même » ${ }^{(4)}$.

Si la mythanalyse n'a pas été définie originellement pour des textes « littéraires », il est bien évident qu'elle s'est appliquée à ceux-ci dès l'origine car les mythes nous parviennent toujours enrobés de littérature (l'exemple d'Edipe traité par LéviStrauss se passe de commentaires). La mythanalyse ne s'intéresse toutefois pas à la littérarité elle-même mais à la substance première ( « mythique ») qui s'est incarnée ou « régénérée » dans une forme littéraire.

\section{Le structuralisme anthropologique}

Il convient à présent de souligner l'apport durandien à la mythanalyse de LéviStrauss. Gêné par le soupçon du non-sens qui pèserait sur le mythe et par la négation supposée du « sujet» humain dans l'idéologie structuraliste, G. Durand radicalise les thèses lévi-straussiennes sur la «pensée sauvage» pour repenser toute l'ontologie de l'être humain. Selon lui, l'imaginaire (nouvelle dénomination de la "pensée sauvage ») ne s'oppose pas à la rationalité mais lui est intimement associé : «Il n'y a pas de coupure entre le rationnel et l'imaginaire, le rationalisme n'étant plus, parmi bien d'autres, qu'une structure polarisante particulière du champ des images. Dès lors, l'on peut assimiler la totalité du psychisme, dès qu'il décolle de l'immédiate sensation, à l'imaginaire et la pensée en sa totalité se trouve intégrée à la fonction symbolique ». L'héritage bachelardien est ici manifeste. Il en découle que l'imaginaire est attaché à une sémantique spécifique fournie par ce que Durand appelle «le trajet anthropologique, c'est-à-dire l'incessant échange qui existe au niveau de l'imaginaire entre les pulsions subjectives et assimilatrices et les intimations objectives émanant du milieu cosmique et social. » ${ }^{(5)}$

Cependant, récusant l'idée que le mythe puisse être assimilé à un langage et ses composantes symboliques aux phonèmes, Durand avance que la discursivité signifiante du mythe réside dans sa réalité symbolique intrinsèque et non dans l'arbitraire du signe linguistique qui le constitue. Si donc le mythe " contient compréhensivement son propre sens », ce dernier le doit à la présence et à la mise en scène de «sé-

(4) Cl. Lévi-Strauss, « La structure du mythe », op. cit.

(5) G. Durand, Les structures anthropologiques de l'imaginaire, Paris, Dunod, 1992 (11 édition), p. 38 . 
quences » de mythèmes qu'il définit comme les plus petites unités sémantiques d'un mythe. Plutôt que de parler, comme le faisait Lévi-Strauss, de « paquets de relations », il préfère parler alors de « paquets de significations ». Pour Durand, la phonologie qui sert de base au structuralisme de Lévi-Strauss manipule des systèmes abstraits ne tenant pas compte des contenus. Elle repose sur trois postulats repris par Lévi-Strauss : les phénomènes qu'on étudie sont inconscients; la conscience est vide donc seuls importent les rapports différentiels ; la phonologie ne s'intéresse pas à la sémantique. Appréciant la déductibilité idéale de la phonologie, Lévi-Strauss extrapole un tel système à l'ensemble du savoir anthropologique (mythologie, ethnologie, etc.) et même à la musique. Or, pour Durand, une structure n'est pas vide, ce que confirme l'origine architecturale du terme «structure » (du latin struere). $\mathrm{Au}$ structuralisme formel, Durand oppose une structure plus concrète qui tient compte des "contenus »; il définit alors la structure comme un système en tension antagoniste de forces (cette définition serait aussi valable pour la chimie, la physique ou les sciences humaines). Le symbole est toujours en tension entre le symbolisant et le symbolisé (l'un pouvant être plus important que l'autre et vice versa). Il est le cœur du jeu des sens (et non du sens) dans les formes.

Produisant les concepts de «mythocritique» et de «mythanalyse», G. Durand applique sa méthode d'étude de l'imaginaire à l'analyse des textes littéraires ou des œuvres artistiques en général (mythocritique) comme il le fait dans Le Décor mythique de La Chartreuse de Parme ${ }^{(6)}$ ou encore à l'analyse des mythes qui "obsèdent » les sociétés. C'est proprement la mythanalyse telle qu'elle se déploie dans Figures mythiques et visages de l'œuvre ${ }^{(7)}$. L'observation des faisceaux de mythes, images et symboles se trouve au centre de toute la démarche mythanalytique.

La caractéristique première du discours mythique est pour Durand (comme pour Lévi-Strauss) la récurrence de ses éléments constituants. C'est par la redondance de ses mythèmes que le mythe se constitue identitairement et qu'il se fait se reconnaître dans sa permanence ou dans ses évolutions. Assimilable à une véritable fonction, le mythème occupe donc une position active au sein du mythe et peut, à ce titre, se repérer dans un "décor », un personnage, une situation. C'est donc le mythème que Durand proposera comme base de sa méthode d'analyse. Trois temps sont distingués lors de l'analyse :

1) Il s'agit tout d'abord d'établir un relevé des séquences ou mythèmes redondants qui constituent, selon la perspective structuraliste, la synchronicité mythique d'un mythe idéal, synthèse de plusieurs versions où se repéreraient des permanences et des invariants. Par exemple, en ce qui concerne le mythe de Don Juan, le défi dans ses différentes déclinaisons (à l'autorité, à Dieu), la séduction féminine, l'attrait pour la mort, le couple maître/valet...

2) Le deuxième temps s'intéresse plutôt à la diachronicité du mythe, c'est-à-dire à la façon dont les différentes séquences de celui-ci se combinent et s'articulent dans un texte ou sur une période donnée. Ainsi, selon la place ou la mise en scène insistante de tel ou tel mythème, c'est toute l'interprétation du mythe étudié ainsi que celle de son contexte qui seront modulées. L'étude d'un mythe repose sur sa « réception ${ }^{(8)}$ à différentes étapes de l'histoire des cultures.

(6) G. Durand, Le Décor mythique de la Chartreuse de Parme, Paris, José Corti, 1961.

(7) G. Durand, Figures mythiques et visages de l'ouvre. De la mythocritique à la mythanalyse, Paris, Berg International, 1979.

(8) H. R. Jauss, Pour une esthétique de la réception, Paris, Gallimard, 1978 ou coll. Tel, 1990. 
3) C'est d'ailleurs précisément l'objet de la troisième étape que de s'interroger sur les différentes versions ou variations d'un mythe. La mythocritique comme la mythanalyse constituent une herméneutique au service du sens intrinsèque du mythe et de l'espace artistique ou social dans lequel il se déploie. Le mythème, « la plus petite unité de discours mythiquement significative " en représente son instrument d'analyse premier.

Ainsi, que les mythèmes soient «patents » c'est-à-dire manifestes, explicites dans leur contenu ou qu'ils soient « latents », c'est-à-dire présents de façon oblique, voire symbolique, le mythe en sera consolidé ou plus ou moins vidé de son sens . C'est pourquoi Durand peut aller jusqu'à fixer la quantité de mythèmes nécessaires à l'identité d'un mythe considéré.

\section{L'idéalisme mythocritique}

La perspective quantitative adoptée par Durand pour statuer sur l'existence et la densité suffisante du mythe dans une œuvre ou sur une période donnée ne rendait pas suffisamment compte de la présence du mythe et de sa signification dans le texte littéraire. C'est pourquoi Pierre Brunel, auteur en 1992 de Mythocritique, théories et parcours $^{(9)}$ s'interroge de manière plus souple et qualitative sur le pouvoir d'« irradiation » du mythe dans l'œuvre d'un auteur. Il préconise que la présence d'un élément mythique d'un texte soit seule considérée comme déterminante et essentiellement signifiante. Pour Brunel, quel que soit le poids du mythe dans un texte, quel que soit le nombre de ses mythèmes présents, c'est toujours à partir de l'élément mythique émergent que doit s'organiser l'analyse. Ainsi en va-t-il de l'épigraphe de la deuxième partie d'Aurélia de Gérard de Nerval, «Eurydice ! Eurydice ! » ou des références allusives au mythe d'Edipe dans le roman d'Alain Robbe-Grillet Les Gommes. Travaillant sur une matière essentiellement littéraire, Brunel a rappelé (à juste titre) l'exigence d'analyse de la lettre dans la réécriture des mythes mais pas nécessairement dans une perspective psychanalytique car celle-ci relève de l'herméneutique réductive que dénonçait Durand. Qu'elle parte de l'analyse du contenu ou de la forme de son expression, l'étude des mythes est souvent tiraillée entre des postulations inconciliables.

Considérés d'une perspective théorique (anthropologique) ou créatrice (littéraire), les mythèmes apparaissent incontestablement et de façon stable comme les unités structurantes du mythe ; ils s'imposent surtout comme les vecteurs d'une sémantique symbolique propre qui ne se réduit ni à l'anthropologie ni à la littérature. Ceux-ci fondent à la fois la création, l'interprétation et la réception des œuvres et témoignent par là même de l'histoire du mythe, c'est-à-dire de son enrichissement ou, à l'opposé, de la perte de son sens.

Certes, la mythocritique de Brunel reste plus ancrée dans la littérarité. Elle répond à la question : comment la littérature se construit-elle « autour» du mythe ou à partir de lui ? Comme le mythe est toujours déjà là, elle vise la compréhension de l'œuvre de l'intérieur pour ainsi dire (et non à partir de l'extériorité d'un mythe hérité). Elle cherche à connaître le cheminement créateur du cogito de l'écrivain confronté doublement à la matérialité du langage et à la substance imaginaire du mythe.

(9) P. Brunel, Mythocritique. Théorie et parcours, Paris, P.U.F., 1992. 
Au contraire, la mythanalyse (ou analyse en constituants mythémiques) suppose la mise en évidence d'une « grammaire de l'imaginaire » qui ne repose pas sur la logique rationnelle (et isolée) d'un cogito pensant et créateur mais sur « l'a-logique du mythe » (G. Durand) et sur un trajet anthropologique. Elle renvoie donc à une bonne connaissance des phénomènes mythiques dans l'ensemble des sociétés. Elle vise la connaissance d'une autre «logique » de la création que celle qui émanerait du cogito d'un écrivain isolé. Elle cherche à comprendre comment toute création individuelle est travaillée en permanence par une attente collective ; aussi est-il vain de se demander qui, un jour, a pu « créer » un mythe. Le grand écrivain est celui qui a su répondre à l'horizon d'attente d'une société en captant dans une œuvre les mythes latents mais actifs de cette société.

\section{Le statut verbo-iconique de l'imaginaire}

A s'en tenir aux développements de la mythanalyse lévi-straussienne, le basculement de l'analyse vers la forme linguistico-mathématique des mythes a fait parfois oublier leur contenu. En 1962, Lévi-Strauss et Jakobson publiaient une célèbre étude « structurale» sur «Les Chats» de Baudelaire ${ }^{(10)}$. En 1969, Durand leur répliquait en proposant une lecture « imaginariste» du même poème. Il y soulignait l'absurdité d'une approche qui ferait abstraction des «sens » en jeu dans les formes syntaxiques, phoniques et métriques repérées par l'analyse structurale. Pour le montrer, Durand propose un pastiche du poème en gardant tous les procédés formels (rimes, parallélismes syntaxiques et phoniques) mis en évidence par ses prédécesseurs mais en changeant le sens de certains mots : "Les amoureux fervents et les savants austères / Aimentégalement dans leur mûre saison / Les rats glissants et mous, orgueil de la prison / Qui comme eux sont miteux et comme eux réfractaires ». Pour l'analyse structurale, le fond de l'analyse lévi-straussienne calée sur la forme ne changerait pas avec ces nouveaux termes, ce qui prouve bien que quelque chose a échappé à l'analyse. Ce « quelque chose » est précisément ce que l'on peut appeler le statut iconique du langage.

L'analyse structurale avait esquivé un obstacle épistémologique de taille : le lien entre signifié, signifiant et référent. Or, ce problème conduit à réévaluer la démarche mythologique dans son ensemble car, si tout n'est pas linguistique dans le mythe, tout n'est pas non plus ethnologique en lui. La mythologie ne peut rester prisonnière d'une ethnologie sans assise linguistique ni d'une philologie sans ouverture ethnoanthropologique. L'enjeu interdisciplinaire est bien là : comment sortir l'analyse mythologique d'une philologie myope et comment marier le structuralisme anthropologique à une sémiologie opérante ?

On ne peut se contenter de juxtaposer les deux démarches en mêlant (plus ou moins habilement) les concepts de l'une et de l'autre. Il faut reposer les données du problème. Dans La vie des images ${ }^{(11)}$, Jean-Jacques Wunenburger apporte une solution originale au dilemme. Sa réponse consiste à valoriser la notion heuristique d'image et à lui donner une existence primordiale dans la vie mentale et l'activité linguistique.

(10) R. Jakobson et Cl. Lévi-Strauss, " "Les Chats” de Charles Baudelaire », L'Homme, 1962, 1, pp. 5-21 (étude accessible en ligne sur le site Persée).

(11) J.-J. Wunenburger, La Vie des Images, Grenoble, P.U.G., 2002, p. 39. 
Lévi-Strauss affirmait que le mythe est « langage » mais qu'il « décolle ensuite du fondement linguistique où il avait commencé par rouler». Mais vers quoi le mythe s'envole-t-il ? Lévi-Strauss ne le précisait pas. En isolant le statut «verbo-iconique » de l'imaginaire et a fortiori du mythe conçu comme « récit d'images », Wunenburger souligne, en bon bachelardien, que l'imaginaire mythique n'est pas réductible au linguistique mais qu'il procède simultanément du verbal et de l'iconique. Selon lui, «l'autonomisation de la substance langagière a conduit à minorer ou à refouler la dimension d'expérience visuelle inhérente aux faits de langage. Or, comme l'a fermement illustré Gaston Bachelard, une image apparaît bien comme " un complexe indissociable d'éléments langagiers (phonétiques, sémantiques) et visuels ».

En d'autres termes, tout n'est pas linguistique dans la pensée et le langage. D'une part, la pensée ne se réduit pas au langage. D'autre part, il existe une «pensée » sans mots, une pensée en images (précédant le langage). L'activité onirique en serait le meilleur exemple dans son état psychique primordial. Les images «mentales » (qui ne sont pas picturales mais mnésiques) forment la matrice de nos pensées. Elles se diversifient ensuite en textes poétiques (ou non), en théorèmes mathématiques ou en réalisations techniques (les Grecs disaient avoir inventé la pince du forgeron après avoir vu celles du crabe). Ce néo-bachelardisme gagnera un jour à être confronté aux acquis des neurosciences. Il n'est pas vain de prophétiser que les possibilités ouvertes actuellement par l'imagerie cérébrale (IRM ou magnétoencéphalographie) se trouveront au cœur de grandes découvertes pour le XXI ${ }^{\mathrm{e}}$ siècle. C'est un fait : on est déjà en mesure de cartographier et disséquer l'activité d'imagerie mentale précédant les actes de langage. Des progrès spectaculaires peuvent résulter de ces observations neurolinguistiques. Pour l'instant, tant qu'il résiste à toute forme de réductionnisme (linguistique, sémiotique, psychanalytique ou autre), l'imaginaire reste un champ ouvert nécessitant le croisement de savoirs multiples profondément dissociés dans le champ des disciplines actuelles. Il reste encore à envisager l'apport de l'informatique à ces nouvelles perspectives.

\section{Le monde fractal}

De Cl. Lévi-Strauss à G. Durand, la théorie de l'imaginaire en est restée à une étape pré-informatique de la recherche. Ce qui frappe, c'est qu'elle n'a pas encore accompli sa révolution numérique. Certes, l'état de l'informatique à l'ère du structuralisme naissant ne permettait pas encore des applications expérimentales concluantes à un corpus de mythes ou d'images (dans une informatique fondée sur l'indexation et les concordances, il n'était pas possible de dépasser le stade de l'observation statistique). Aujourd'hui, il n'en est plus de même. L'analyse des images visuelles par l'informatique devient possible car les progrès de l'analyse numérique apportent des outils heuristiques nouveaux pour créer ces images (films 3D) et pour les analyser. Il reste à se demander à quelles règles obéit la transformation généralisée (morphogénèse) des images. Les images visuelles ont-elles le même statut que les images verbales ? Sur ce point, Durand et Wunenburger sont réservés. La grammaire de l'imaginaire ne s'inscrit pas dans le paradigme d'une logique des «catégories » (linguistiques, syntaxiques ou autres) mais plutôt d'une analyse des « catégories de l'irrationnel ». Nous dirions plutôt d'une anthropo-logique « fractale » (Benoît Mandelbrot).

Toute «création », une concrétion naturelle (on songe naturellement aux ré- 
flexions de Roger Caillois sur les pierres) comme une œuvre d'art, résulte de procédures créatrices complexes voire « chaotiques » (R. Thom) qu'il est possible d'étudier grâce à la reconnaissance d'homothéties internes, incluant un mécanisme général de répétition (la « récurrence» du mythe ou le symptôme freudien) et une dimension fractale. Selon la formule de Mandelbrot, dans un objet fractal, « le détail reproduit la partie et la partie reproduit le tout». Or, c'est exactement une qualité que Durand reconnaît au mythe en le définissant comme hologramme. La théorie des fractales permet de formaliser des lois d'autosimilarité qui jouent aussi bien dans le fractionnement des motifs mythiques d'un texte, l'iconicité d'un tableau ou la «sérialité » sonore d'une œuvre musicale.

Parmi les acquis récents de la réflexion méthodologique au sein du CRI de Grenoble, la reconnaissance de la nature fractale des phénomènes de l'imaginaire paraît féconde. Cette dimension, jadis approchée par G. Durand à travers le concept d'hologramme, semble se décliner aujourd'hui avec une plus grande fécondité heuristique dans le concept mathématique de « monde fractal». Les fractales constituent une technique de représentations mathématique récente développée par le mathématicien français Mandelbrot depuis les années 1970. L'intuition en existait dès la fin du $\mathrm{XIX}^{\mathrm{e}}$ siècle sous la dénomination de «monstres mathématiques ». Pour la mathématique euclidienne, une orange et une boule de billard se ramènent à une sphère. Pour les fractales, ces deux objets sont nettement différenciés par leur état de surface et nécessitent des modèles explicatifs différents.

Les fractales décrivent des structures complexes (par exemple, un flocon de neige) à partir de fractions (d'où le mot fractal) de segments récurrents. Des homothéties internes, répétition à différentes échelles de formes simples, sont reproductibles à l'aide de l'ordinateur qui devient un outil essentiel d'analyse des formes. Autrement dit, le mythe se définit fondamentalement par la récurrence de ses éléments constituants mais cette récurrence est jusqu'alors perçue de manière aléatoire et statistique. Il y a en fait dans les récits mythiques ou l'œuvre d'art les mêmes principes d'homothétie (fractale) que dans les formes et objets du monde visible. La théorie mathématique des fractales fournit aujourd'hui des outils (algébriques, géométriques ou algorithmiques) pour modéliser les modalités de la récurrence des mythèmes. Il n'est pas possible, dans le cadre de cet essai, d'apporter une démonstration détaillée de l'ensemble de la démarche dont les différents paramètres sont en cours de traitement informatique. Toutefois, en reprenant l'exemple des Chats de Baudelaire, il est possible de comprendre comment se croisent la dimension verbale et la dimension iconique du texte. Visuellement, le poème repose sur une image de chat qui se transforme en sphinx. Parallèlement, il se développe à partir d'une cellule phonétique /chat/ en métamorphose constante grâce aux homophonies et variations de phonèmes. Dans les deux premiers vers du poème, la dentale pré-dorso alvéolaire de /chat/ allitère in absentia à travers d'autres sons de la même classe phonologique des sibilantes dans la série $\mathrm{S} \ldots \mathrm{Z} \ldots \mathrm{Z} \ldots \mathrm{S}$, la dentale sifflante apico-alvéolaire sonore $\mathrm{Z}$ et la sourde $\mathrm{S}$ :

« Les amoureux fervents et les savants austères

Aiment également, dans leur mûre saison,

Les chats puissants et doux, orgueil de la maison,

Qui comme eux sont frileux et comme eux sédentaires. »

Et ainsi de suite jusqu'à la fin du poème. Le poème repose ainsi sur la transformation de matrices verbales et iconiques en interaction constante pour le son et l'image 
suscitée par les sons. Paul Valéry parlait à juste titre de l'hésitation du son et du sens constitutive de la création poétique. L'émergence de la figure mythique du sphinx au centre du poème est préparée par un jeu de matrices sonores et iconiques en métamorphose constante ; elles développent par ailleurs la transformation visuelle du chat en sphinx, selon des procédures qui restent à décrire mais qui ne relèvent pas toutes de la rationalité car la rupture et l'analogie ont toute leur part dans ce processus créateur :

«Ils prennent en songeant les nobles attitudes

Des grands sphinx allongés au fond des solitudes,

Qui semblent s'endormir dans un rêve sans fin (...).»

Les sciences cognitives peuvent apporter à cette description une contribution intéressante. Les processus d'association d'idées, les phénomènes d'illusion perceptive (sonore ou visuelle) ont leur part dans cette morphogénèse des images. En effet, si l'imagination est la capacité de former des images, l'imaginaire est la capacité de les déformer et de les transformer, autrement dit (sur le plan des images visuelles) de quitter les formes simples (cercle, ligne, carré) pour s'orienter vers les formes complexes (que la théorie des fractales peut décrire).

Keiji Tanaka, un neurophysiologiste japonais, avait découvert dès 1990 dans le cerveau des singes une sorte de «dictionnaire cortical de formes élémentaires ». Il a montré que les neurones de leur cortex temporal inférieur peuvent coder toutes les formes élémentaires retrouvées dans la nature. Certains neurones de cette zone répondent préférentiellement à la perception de deux barres formant un $\mathrm{T}$, par exemple, d'autres préfèrent les formes en Y, d'autres encore les formes en 8 . Autour de nous, lorsque les objets perçus se superposent, leurs contours font apparaître des jonctions en forme de T le plus souvent, mais aussi en L, en Y, etc. Combinables entre elles, ces formes élémentaires codées dans notre cerveau constitueraient un véritable alphabet permettant de décrire n'importe quel objet. L'utilisation des fractales est un outil permettant de décrire de telles combinatoires. En tout cas, cet alphabet neuronal se serait constitué en partie au cours de l'évolution mais se développerait surtout lors de l'apprentissage visuel de l'enfant. Avant même d'apprendre à lire ou à écrire, nous posséderions ces « proto-lettres » (selon l'expression de S. Dehaene). L'étude du graphisme dans l'art rupestre de la préhistoire conduit à des observations convergentes.

Il existe parallèlement pour les textes (et les mots) des modèles connus de déformation/transformation. Il s'agit des figures de rhétorique. Figure (de figura) désigne la forme. En grec, la figure de rhétorique se dit skema, autrement dit "schème », ou «schéma ». Posons alors qu'une figure de rhétorique est le modèle verbal d'une figure ou d'une forme qui peut avoir des correspondances «schématiques » visuelles. Un mot est aussi un geste (visuel et mental). Remarquons ici que la rencontre du verbal et de l'iconique s'opère dans les systèmes d'écriture car ceux-ci exploitent les proto-lettres de la vision et les transposent en symboles écrits du langage parlé. Il faudrait d'ailleurs se demander si tous les systèmes d'écriture utilisent les mêmes proto-lettres.

C'est donc bien en direction des sciences cognitives que les recherches sur l'imaginaire trouveront une voie d'avenir. Celle-ci était déjà tracée par ses orientations initiales. Selon G. Durand en effet, " l'Imaginaire - c'est-à-dire l'ensemble des images et des relations d'images qui constitue le capital pensé de l'homo sapiens - nous 
apparaît comme le grand dénominateur fondamental où viennent se ranger toutes les procédures de la pensée humaine ». Autant dire que nous n'en sommes qu'au début d'une longue et passionnante aventure de l'esprit. Plus que jamais la science de l'homme apparaît comme « une science des confins de la connaissance ${ }^{(12)}$.

(12) Expression employée pour la première fois par G. Durand lors du colloque de l'UNESCO (Venise, 3-7 mars 1986) dans sa conférence : «La science de l'homme : science des confins de la connaissance ». Texte consultable sur Internet : http://unesdoc.unesco.org/images/006/000678/067819fb.pdf/. On signalera également l'ouvrage récent: Y. Durand, J.P. Sironneau et A.F. Aranjo, eds., Variations sur l'imaginaire. L'épistémologie ouverte de G. Durand. Orientations et innovations, Bruxelles, E.M.E., 2011.

\section{LE RANCAIS AUJOUR̉D'HUI}

\section{CONTINUITES ET RUPTURES DANS L'ENSEIGNEMENT DE LA LANGUE}

Numéro coordonné par

Marie-France Bishop et Lucile Cadet

Contributions de

Bernard Combettes

Interroger l'enseignement de la langue

Gérard Vigner, Marie-Laure Elalouf, Danièle Cogis, Patrice Gourdet, Suzanne Chartrand

Analyser les textes officiels et les supports d'apprentissage

Emmanuelle Guerin, Françoise Bollengier, Isabelle de Peretti

Travailler la langue dans d'autres disciplines

Marc Debono, Sophie Bigot de Préameneu

Postface

Claude Vargas

Chroniques

Serge Martin, Christine Plu

Revue de l'Association Française des Enseignants de Français

Courriel editeur : infos@armand-colin.fr

Courriel rédaction : le-francais-aujourdhui@wanadoo.fr

$\mathrm{N}^{\circ} 173$ - Juin 2011 - ISBN 978-2-200-92711-0 